\title{
Crystallographic disorder of carbonyl, propyl isocyanide and acetonitrile groups in the structures of $\mathrm{Os}_{3}(\mathrm{CO}){ }_{10}(\mathrm{CNR})(\mathrm{NCMe})$
}

\author{
Kuang-Lieh Lu a, Ling-Kang Liu ${ }^{\text {a,b }}$, Chi-Jung Su ${ }^{\text {a,c }}$, Han-Mou Gau ${ }^{c}$, Yu Wang ${ }^{\text {b }}$ \\ and Gene-Hsiang Lee ${ }^{b}$ \\ ${ }^{a}$ Institute of Chemistry, Academia Sinica, Taipei (Taiwan) \\ ${ }^{b}$ Department of Chemistry, National Taiwan University, Taipei (Taiwan) \\ ' Department of Chemistry, National Chung-Hsing University, Taichung (Taiwan)
}

(Received December 15, 1993; in revised form March 2, 1994)

\begin{abstract}
Crystallographic disorder between structurally inequivalent groups in complexes $\mathrm{Os}_{3}(\mathrm{CO})_{10}(\mathrm{CNR})(\mathrm{NCMe})(1 \mathrm{a}, \mathrm{R}=\mathrm{Ph} ; \mathbf{1 b}, \mathrm{R}=\mathrm{Pr})$ has been investigated by X-ray structure analysis. The disorder between NCMe and $\mathrm{CO}$ in $1 \mathrm{a}$ is caused by a crystallographic mirror symmetry whercas the disorder between NCMe and CNPr in $1 \mathrm{~b}$ is caused by a crystallographic two-fold symmetry. Crystal data: 1a: $P 2_{1} / m ; a=8.047(2), b=12.125(3), c=12.549(2) \AA ; \beta=106.50(2)^{\circ}, V=1174.0(5) \AA^{3}, Z=2, R=0.037, R_{w}=0.029 .1 \mathrm{~b}: P b c n$; $a=13.292(1), b=12.655(2), c=14.926(2) \AA ; \quad V=2510.6(5) \AA^{3}, Z=4, R=0.045, R_{w}=0.046$.
\end{abstract}

Key words: Osmium; Acetonitrile; Carbonyl; Crystallography; Disorder; Isocyanide

\section{Introduction}

Crystallographic disorder between structurally inequivalent groups is well known [1]. Many examples have been observed of disorder between carbonyl and other ligand [2], $\mathrm{N}_{2}$ and $\mathrm{Cl}$ [3], methyl $\left(\mathrm{CH}_{3}\right)$ and ethylidyne $\left(\mathrm{C} \equiv \mathrm{CH}_{3}\right)[4]$ in metal complexes, however, disorder between coordinated NCMe and other groups is less thoroughly explored. In the course of previous attempts to learn the effect of isocyanide-carbonyl replacement on the reactivity of the metal carbonyl cluster, we studied the preparation and reactivity of the "lightly stabilized" complexes $\mathrm{Os}_{3}(\mathrm{CO})_{10}(\mathrm{CNR})$ (NCMe) [5]. Here we report our findings on the characterization and comparison of molecular structures of $\mathrm{Os}_{3}(\mathrm{CO})_{10}(\mathrm{CNR})(\mathrm{NCMe})(\mathbf{1 a}, \mathrm{R}=\mathrm{Ph} ; \mathbf{1 b}, \mathrm{R}=\mathrm{Pr})$. The NCMe ligand is disordered with $\mathrm{CO}$ in complex 1a; whereas NCMe is disordered with CNPr group in $\mathbf{1 b}$. These observations provided examples of disordered

Correspondence to: Dr. K.-L. Lu or Dr. L.-K. Liu. crystal structure between the NCMe, $\mathrm{CO}$ and CNR ligands, differing in both composition and structure.

\section{Results and discussion}

\subsection{Crystal structure of $\mathrm{Os}_{3}(\mathrm{CO})_{10}(\mathrm{CNPh})(\mathrm{NCMe})$}

The molecular structure and atomic labelling scheme of $\mathrm{Os}_{3}(\mathrm{CO})_{10}(\mathrm{CNPh})(\mathrm{NCMe})(1 \mathrm{a})$ are shown in Fig. 1. The three Os atoms define an isosceles triangle. The CNPh ligand occupies an axial coordination site on Os(1). The NCMe ligand is coordinated at the axial site of $\mathrm{Os}(2)$ but disordered with the axial $\mathrm{CO}$ ligand $[C(6) O(6)]$ as required by crystallographic mirror symmetry. Both the NCMe and $\mathrm{C}(6) \mathrm{O}(6)$ ligands are anti to the $\mathrm{CNPh}$ ligand with respect to a plane defined by three Os atoms. The NCMe group and the [C(6)O(6)] group have been successfully and independently refined. The bond length of Os(2)-C(6) [1.73(3) $\AA]$ is shorter than the other axial bond lengths of Os(2)-C$[1.87(2), 1.86(1) \AA]$ and that of $C(6)-O(6)[1.29(4) \AA]$ is longer than other carbonyls [average $\mathrm{C}-\mathrm{O}=1.16(2) \AA$ ] in the molecule, whereas the Os-N(2) [2.16(1) $\AA]$ is 


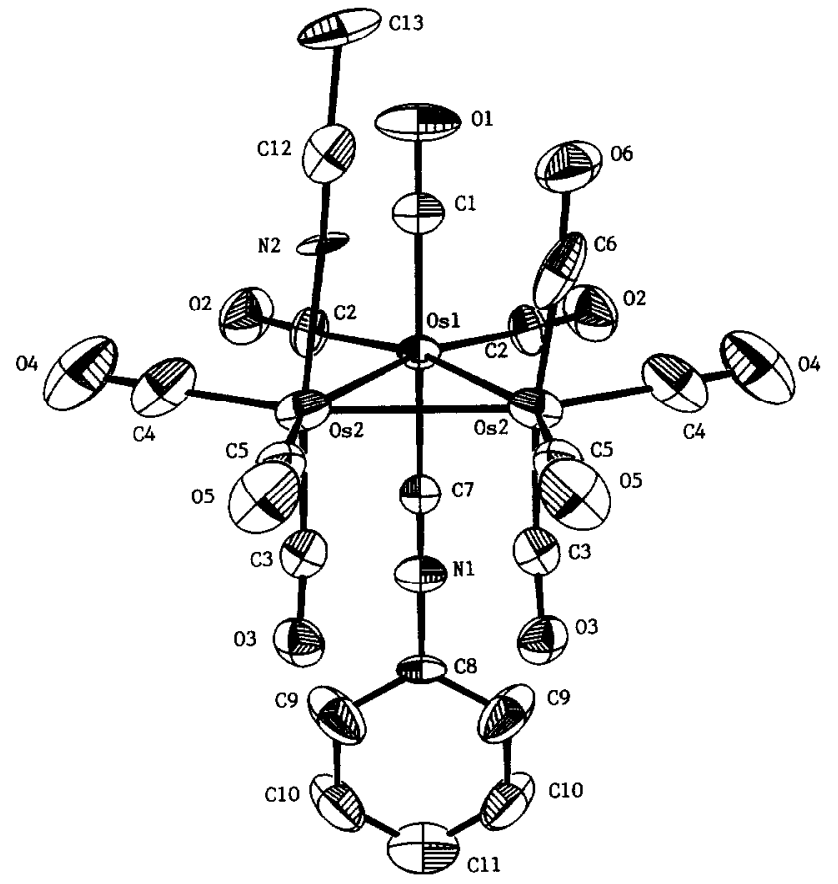

Fig. 1. ORTEP diagram of $\mathrm{Os}_{3}(\mathrm{CO})_{10}(\mathrm{CNPh})(\mathrm{NCMe})(1 \mathrm{a})$. Only one site for the NCMe ligand and $\mathrm{C}(6) \mathrm{O}(6)$ group is shown. longer than that of the related acetonitrile complexes $\mathrm{Os}_{3}(\mathrm{CO})_{11}(\mathrm{NCMe})[2.07(2) \AA]$ and $\mathrm{Os}_{3}(\mathrm{CO})_{10}(\mathrm{NCMe})_{2}$ $[2.13(2), 2.13(1) \AA][6]$. These structural parameters are only known with limited accuracy, resulting from incomplete separation of electron density peaks into $\mathrm{N}(2)$ and $\mathrm{C}(6)$, together with poorly defined anisotropic thermal parameters. The axial Os- $\mathrm{C}$ distances are apparently shorter than the equatorial Os $-\mathrm{C}$ lengths. The vectors of $\mathrm{Os}(2)-\mathrm{NCMe}$ and $\mathrm{Os}(2)-\mathrm{C}(6) \mathrm{O}(6)$ are not truly coincident $\left[\mathrm{N}(2)-\mathrm{Os}(2)-\mathrm{C}(6)=19(1)^{\circ}\right]$.

\subsection{Crystal structure of $\mathrm{Os}_{3}(\mathrm{CO})_{10}(\mathrm{CNPr})(\mathrm{NCMe})$}

Replacing CNPh by a less symmetric CNPr ligand, $\mathrm{X}$-ray crystal structure analysis of complex $\mathrm{Os}_{3}(\mathrm{CO})_{10^{-}}$ (CNPr)(NCMe) (1b) was undertaken in order to see to what extent the removal of the (disorder-causing) crystallographic mirror perpendicular to the $\mathrm{Os}_{3}$ plane would affect the crystal structure. An ORTEP drawing of 1b is shown in Fig. 2. Interestingly, its crystal structure also possesses a disorder problem yet in a different pattern. Three Os atoms define a triangle skeleton with similar Os-Os bond lengths. Os(1) is coordinated by four $\mathrm{CO}$ groups. The CNPr and NCMe ligands are coordinated at the axial site of the other two Os atoms, respectively, and anti to each other with respect to the

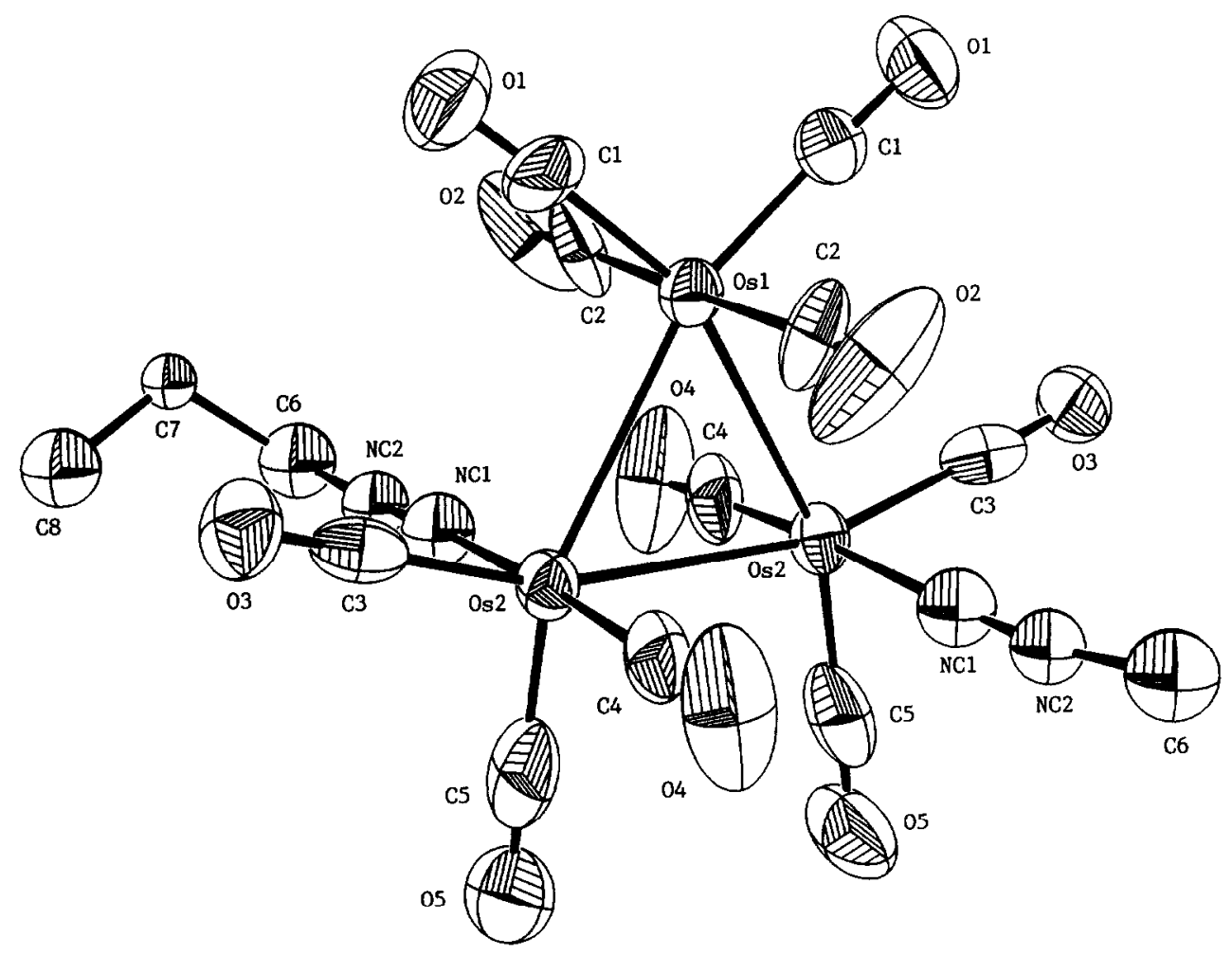

Fig. 2. ORTEP diagram of $\mathrm{Os}_{3}(\mathrm{CO})_{10}(\mathrm{CNPr})(\mathrm{NCMe})(\mathbf{b b})$. 
TABLE 1. Crystal and intensity collection data for $\mathrm{Os}_{3}(\mathrm{CO})_{10^{-}}$ $(\mathrm{CNPh})(\mathrm{NCMe})(1 \mathrm{a})$ and $\mathrm{Os}_{3}(\mathrm{CO})_{10}(\mathrm{CNPr})(\mathrm{NCMe})(\mathrm{bb})$

\begin{tabular}{lll}
\hline & $1 \mathrm{a}$ & $\mathbf{1 b}$ \\
\hline formula & $\mathrm{C}_{18} \mathrm{H}_{8} \mathrm{~N}_{2} \mathrm{O}_{10} \mathrm{Os}_{3}$ & $\mathrm{C}_{16} \mathrm{H}_{8} \mathrm{~N}_{2} \mathrm{O}_{10} \mathrm{Os}_{3}$ \\
fw & 985.89 & 914.87 \\
space group & $P 2_{1} / m$ & $P b c n$ \\
$a, \AA$ & $8.047(2)$ & $13.292(1)$ \\
$b, \AA$ & $12.125(3)$ & $12.655(2)$ \\
$c, \AA$ & $12.549(2)$ & $14.926(2)$ \\
$\beta$, deg & $106.50(2)$ & \\
$V, \AA^{3}$ & $1174.0 \times 5)$ & $2510.6(5)$ \\
$D_{\text {calc }}, \mathrm{g} \mathrm{cm}^{-3}$ & 2.789 & 2.420 \\
$Z$ & 2 & 4 \\
cryst dimension, mm & $0.32 \times 0.32 \times 0.40$ & $0.20 \times 0.20 \times 0.20$ \\
abs coeff $\mu(\mathrm{MoK} \alpha), \mathrm{mm}^{-1}$ & 16.27 & 15.21 \\
temp & room temperature & room temperature \\
radiation & $\mathrm{MoK} \alpha$ & $\mathrm{MoK} \alpha$ \\
$2 \theta$ (max) & $49.9^{\circ}$ & $49.8^{\circ}$ \\
scan type & $\theta / 2 \theta$ & $\theta / 2 \theta$ \\
total no. of reflctn & 2329 & 2231 \\
no. of observed reflctn & 1629 & 1073 \\
$F_{o}>2 \sigma\left(F_{o}\right)$ & & \\
observed variables & 188 & 121 \\
$R$ & 0.037 & 0.045 \\
$R_{w}$ & 0.029 & 0.046 \\
$\Delta(\rho)$, e $\AA^{-3}$ & 1.290 & 1.290 \\
$\Delta / \sigma_{\text {max }}$ & 0.062 & 0.035 \\
GOF & 2.50 & 2.59 \\
\hline & & \\
\hline
\end{tabular}

TABLE 2. Atomic coordinates and isotropic thermal parameters $\left(\AA^{2}\right)$ for $\mathrm{Os}_{3}(\mathrm{CO})_{10}(\mathrm{CNPh} X \mathrm{NCMe})(1 \mathrm{a})$

\begin{tabular}{|c|c|c|c|c|}
\hline$\overline{\text { Atom }}$ & $x$ & $y$ & $\bar{z}$ & $B_{\text {iso }}{ }^{a}$ \\
\hline Os1 & $0.50259(9)$ & $1 / 4$ & $0.37024(6)$ & 3.14(3) \\
\hline Os2 & $0.23018(7)$ & $0.13214(5)$ & $0.21566(4)$ & $4.55(3)$ \\
\hline N1 & $0.3029(17)$ & $1 / 4$ & $0.5514(10)$ & $4.3(8)$ \\
\hline N2 & $0.3597(21)$ & $0.1552(15)$ & $0.0884(13)$ & $3.4(9)$ \\
\hline $\mathrm{C} 1$ & $0.6289(20)$ & $1 / 4$ & $0.2660(12)$ & $3.7(9)$ \\
\hline C2 & $0.6284(17)$ & $0.1282(10)$ & $0.4460(9)$ & $4.6(7)$ \\
\hline $\mathrm{C} 3$ & $0.1056(14)$ & $0.1299(10)$ & $0.3203(9)$ & $3.9(6)$ \\
\hline $\mathrm{C} 4$ & $0.3109(16)$ & $-0.0142(13)$ & $0.2523(11)$ & $7.0(9)$ \\
\hline C5 & $0.0139(16)$ & $0.1160(11)$ & $0.0976(11)$ & $5.3(8)$ \\
\hline C6 & $0.361(4)$ & $0.1080(23)$ & $0.1297(22)$ & $6.9(16)$ \\
\hline C7 & $0.3712(19)$ & $1 / 4$ & $0.4802(11)$ & $2.9(8)$ \\
\hline $\mathrm{C} 8$ & $0.2172(20)$ & $1 / 4$ & $0.6354(13)$ & $3.9(10)$ \\
\hline $\mathrm{CP}$ & $0.1750(23)$ & $0.3464(13)$ & $0.6752(12)$ & $10.0(13)$ \\
\hline $\mathrm{C} 10$ & $0.0946(23)$ & $0.3476(13)$ & $0.7592(12)$ & $9.4(12)$ \\
\hline $\mathrm{C} 11$ & $0.0456(30)$ & 0.2500 & $0.7977(16)$ & $8.4(17)$ \\
\hline $\mathrm{C} 12$ & $0.4239(29)$ & $0.1628(21)$ & $0.0189(20)$ & $5.0(13)$ \\
\hline $\mathrm{C} 13$ & $0.5166(36)$ & $0.1751(25)$ & $-0.0692(20)$ & $7.6(20)$ \\
\hline O1 & $0.7152(16)$ & 0.2500 & $0.2046(9)$ & $7.5(10)$ \\
\hline $\mathrm{O} 2$ & $0.7107(13)$ & $0.0541(8)$ & $0.4901(8)$ & $8.1(7)$ \\
\hline $\mathrm{O3}$ & $0.0237(10)$ & $0.1232(7)$ & $0.3838(6)$ & $5.1(5)$ \\
\hline O4 & $0.3634(15)$ & $-0.1032(10)$ & $0.2762(9)$ & $11.3(9)$ \\
\hline 05 & $-0.1082(12)$ & $0.1054(9)$ & $0.0278(7)$ & $7.7(6)$ \\
\hline O6 & $0.4485(28)$ & $0.0995(17)$ & $0.0582(16)$ & $8.7(15)$ \\
\hline
\end{tabular}

\footnotetext{
${ }^{a} B_{\text {iso }}$ is the mean of the principal axes of the thermal ellipsoid.
}

TABLE 3. Selected bond distances and angles for $\mathrm{Os}_{3}(\mathrm{CO})_{10^{-}}$ $(\mathrm{CNPh})(\mathrm{NCMe})(1 \mathrm{a})$

\begin{tabular}{|c|c|c|c|}
\hline \multicolumn{4}{|l|}{ Bond Distances $(\AA)$} \\
\hline$O s(1)-O s(2)$ & $2.8627(10)$ & $N(2)-O(6)$ & $1.12(3)$ \\
\hline $\operatorname{Os}(1)-O s(2)$ & $2.8627(10)$ & $C(1)-O(1)$ & $1.175(19)$ \\
\hline Os(1)-C(1) & $1.870(16)$ & $C(2)-O(2)$ & $1.159(16)$ \\
\hline $\mathrm{Os}(1)-\mathrm{C}(2)$ & $1.888(12)$ & $C(3)-O(3)$ & $1.173(14)$ \\
\hline $\mathrm{Os}(1)-\mathrm{C}(2)$ & $1.888(12)$ & $O(4)-O(4)$ & $1.166(20)$ \\
\hline Os(1)-C(7) & $1.962(15)$ & $C(5)-O(5)$ & $1.123(16)$ \\
\hline $\mathrm{Os}(2)-\mathrm{Os}(2)$ & $2.8579(15)$ & $C(6)-C(12)$ & $1.74(4)$ \\
\hline $\mathrm{Os}(2)-\mathrm{N}(2)$ & $2.158(14)$ & $C(6)-O(6)$ & $1.29(4)$ \\
\hline$O s(2)-C(3)$ & $1.864(11)$ & $C(8)-C(9)$ & $1.351(17)$ \\
\hline Os(2)-C(4) & $1.900(16)$ & $C(8)-C(9)$ & $1.351(17)$ \\
\hline$O s(2)-C(5)$ & $1.949(13)$ & $C(9)-C(10)$ & $1.385(19)$ \\
\hline $\mathrm{Os}(2)-\mathrm{C}(6)$ & $1.73(3)$ & $C(10)-C(11)$ & $1.378(20)$ \\
\hline$N(1)-C(7)$ & $1.175(20)$ & $C(11)-C(10)$ & $1.378(20)$ \\
\hline$N(1)-C(8)$ & $1.414(20)$ & $C(12)-C(13)$ & $1.51(3)$ \\
\hline$N(2)-C(6)$ & $0.77(3)$ & $C(12)-O(6)$ & $0.90(3)$ \\
\hline$N(2)-C(12)$ & $1.14(3)$ & $C(13)-C(13)$ & $1.82(6)$ \\
\hline \multicolumn{4}{|l|}{ Bond Angles (deg) } \\
\hline $\operatorname{Os}(2)-\operatorname{Os}(1)-\operatorname{Os}(2)$ & $59.89(3)$ & $C(7)-N(1)-C(8)$ & $178.8(15)$ \\
\hline $\mathrm{Os}(2)-\mathrm{Os}(1)-\mathrm{C}(1)$ & $89.4(4)$ & $O s(2)-N(2)-C(6)$ & $47(3)$ \\
\hline $\mathrm{Os}(2)-\mathrm{Os}(1)-\mathrm{C}(2)$ & $98.5(4)$ & $\mathrm{Os}(2)-\mathrm{N}(2)-\mathrm{C}(12)$ & $176.7(17)$ \\
\hline $\mathrm{Os}(2)-\mathrm{Os}(1)-\mathrm{C}(2)$ & $158.4(4)$ & $O s(2)-N(2)-O(6)$ & 131.2(17) \\
\hline $\mathrm{Os}(2)-\mathrm{Os}(1)-\mathrm{C}(7)$ & $90.8(4)$ & $C(6)-N(2)-C(12)$ & $131(3)$ \\
\hline Os(2)-Os(1)-C(1) & $89.4(4)$ & $C(6)-N(2)-O(6)$ & $83(3)$ \\
\hline$O s(2)-O s(1)-C(2)$ & $158.4(4)$ & $C(12)-N(2)-O(6)$ & $47.1(17)$ \\
\hline $\mathrm{Os}(2)-\mathrm{Os}(1)-\mathrm{C}(2)$ & $98.5(4)$ & $O s(1)-C(1)-O(1)$ & $176.9(13)$ \\
\hline $\operatorname{Os}(2)-O s(1)-C(7)$ & $90.8(4)$ & $O s(1)-C(2)-O(2)$ & $177.1(11)$ \\
\hline$C(1)-O s(1)-C(2)$ & $91.8(5)$ & $\mathrm{Os}(2)-\mathrm{C}(3)-\mathrm{O}(3)$ & $176.5(10)$ \\
\hline$C(1)-O s(1)-C(2)$ & $91.8(5)$ & $O s(2)-C(4)-O(4)$ & $178.7(12)$ \\
\hline$C(1)-O s(1)-C(7)$ & $179.7(6)$ & $O s(2)-C(5)-O(5)$ & $178.1(11)$ \\
\hline$C(2)-O s(1)-C(2)$ & $103.0(5)$ & $\mathrm{Os}(2)-\mathrm{C}(6)-\mathrm{N}(2)$ & $113(3)$ \\
\hline$C(2)-O s(1)-C(7)$ & $88.0(4)$ & $\mathrm{Os}(2)-\mathrm{C}(6)-\mathrm{C}(12)$ & $143.0(20)$ \\
\hline$C(2)-O s(1)-C(7)$ & $88.0(4)$ & $O s(2)-C(6)-O(6)$ & $173.1(20)$ \\
\hline $\operatorname{Os}(1)-O s(2)-O s(2)$ & $60.056(20)$ & $N(2)-C(6)-C(12)$ & $29.5(21)$ \\
\hline$O s(1)-O s(2)-N(2)$ & $89.9(4)$ & $N(2)-C(6)-O(6)$ & $59.7(25)$ \\
\hline $\operatorname{Os}(1)-O s(2)-C(3)$ & $89.5(3)$ & $C(12)-C(6)-O(6)$ & $30.3(14)$ \\
\hline $\operatorname{Os}(1)-O s(2)-C(4)$ & 99.1(4) & $\mathrm{Os}(1)-\mathrm{C}(7)-\mathrm{N}(1)$ & $175.6(13)$ \\
\hline$O s(1)-O s(2)-C(5)$ & $155.8(4)$ & $N(1)-C(8)-C(9)$ & $120.2(8)$ \\
\hline$O s(1)-O s(2)-C(6)$ & 91.3(8) & $N(1)-C(8)-C(9)$ & $120.2(8)$ \\
\hline $\operatorname{Os}(2)-O s(2)-N(2)$ & $82.6(5)$ & $C(9)-C(8)-C(9)$ & $119.6(14)$ \\
\hline$O s(2)-O s(2)-C(3)$ & $90.9(4)$ & $C(8)-C(9)-C(10)$ & $120.8(14)$ \\
\hline $\operatorname{Os}(2)-O s(2)-C(4)$ & $159.0(4)$ & $C(9)-C(10)-C(11)$ & $120.0(15)$ \\
\hline $\mathrm{Os}(2)-\mathrm{Os}(2)-\mathrm{C}(5)$ & $95.8(4)$ & $C(10)-C(11)-C(10)$ & $118.4(17)$ \\
\hline$O s(2)-O s(2)-C(6)$ & $99.8(9)$ & $N(2)-C(12)-C(6)$ & $19.5(15)$ \\
\hline$N(2)-O s(2)-C(3)$ & $172.7(6)$ & $N(2)-C(12)-C(13)$ & $177.3(24)$ \\
\hline$N(2)-O s(2)-C(4)$ & $95.5(7)$ & $\mathrm{N}(2)-\mathrm{C}(12)-\mathrm{O}(6)$ & $65.8(22)$ \\
\hline$N(2)-O s(2)-C(5)$ & $88.0(6)$ & $C(6)-C(12)-C(13)$ & $160.2(23)$ \\
\hline$N(2)-O s(2)-C(6)$ & 19.1(10) & $C(6)-C(12)-O(6)$ & $46.3(19)$ \\
\hline$C(3)-O s(2)-C(4)$ & $91.8(6)$ & $C(13)-C(12)-O(6)$ & $114(3)$ \\
\hline$C(3)-O s(2)-C(5)$ & $89.5(5)$ & $C(12)-C(13)-C(13)$ & $95.7(17)$ \\
\hline$C(3)-O s(2)-C(6)$ & $168.2(10)$ & $N(2)-O(6)-C(6)$ & $36.3(16)$ \\
\hline$C(4)-O s(2)-C(5)$ & $105.1(5)$ & $N(2)-O(6)-C(12)$ & $67.2(23)$ \\
\hline$C(4)-O s(2)-C(6)$ & $76.5(10)$ & $C(6)-O(6)-C(12)$ & $103(3)$ \\
\hline$C(5)-O s(2)-C(6)$ & $94.5(9)$ & & \\
\hline
\end{tabular}

$\mathrm{Os}_{3}$ plane. The CNPr and NCMe ligands are disordered with each other as related by a crystallographic two-fold axis. 
If there are sufficient voids, a less-symmetric molecule can be packed more freely in lattice to behave on average like a more-symmetric moiety as observed by X-ray crystal structure analysis. For example, an octahedral $\mathrm{PF}_{6}^{-}$anion usually behaves like a sphere in the analysis, such that the $\mathrm{F}$ atoms are characterized with large thermal ellipsoids and often with more than one orientation [7]. In the present study, 1a and 1b have a $C 1$ molecular symmetry and occupy, respectively, crystallographic special positions of mirror and two-fold. Concerning the molecular volumes, $\mathrm{Os}_{3}(\mathrm{CO})_{12}$ has a volume of $427.6 \AA^{3}$ as calculated [8] from its crystallographic cell volume $/ Z ; \mathrm{Os}_{3}(\mathrm{CO})_{11^{-}}$ (NCMe) $476.7 \AA^{3}$; and $\mathrm{Os}_{3}(\mathrm{CO})_{10}(\mathrm{NCMe})_{2} 516.6 \AA^{3}$ [6]. For 1a, the molecular volume is $587.0 \AA^{3}$ within reasonable expansion from the above parent compounds, taking a 15-35 $\AA^{3}$ increase in volume for each additional non-H atom. For $\mathbf{l b}$, the molecular volume is $627.8 \AA^{3}$ which is too big for $1 \mathrm{~b}$ to be packed efficiently in the solid state.

$\mathrm{A} \mathrm{Ph}$ skeleton is generally more dominating towards the crystal packing than a Pr skeleton because the $\mathrm{Ph}$ group is much more rigid due to its planar hexagonal shape. As 1a has a CNPh ligand and 1b has a CNPr ligand, a more efficient packing would be expected for $1 \mathrm{a}$ in which $\mathrm{CO}$ and NCMe do not affect much of the crystallization process, resulting in the mirror imposed disorder. For 1b, the packing is determined partly by the $\mathrm{Os}_{3}(\mathrm{CO})_{10}$ skeleton (23 non-H atoms) which occupies less than ca. $370 \AA^{3}$, (estimated from $427.6 \AA^{3}$ less

TABLE 4. Atomic coordinates and isotropic thermal parameters $\left(\AA^{2}\right)$ for $\mathrm{Os}_{3}(\mathrm{CO})_{10}(\mathrm{CNPr})(\mathrm{NCMe})(\mathbf{1 b})$

\begin{tabular}{llllc}
\hline Atom & $x$ & $y$ & $z$ & $B_{\text {iso }}{ }^{\mathrm{a}}$ \\
\hline Os1 & 0 & $0.53910(14)$ & $1 / 4$ & $5.11(9)$ \\
Os2 & $0.08960(9)$ & $0.34206(11)$ & $0.19659(8)$ & $4.94(6)$ \\
C1 & $0.0917(21)$ & $0.6335(24)$ & $0.1938(22)$ & $8.2(20)$ \\
C2 & $-0.0874(21)$ & $0.5415(26)$ & $0.1388(20)$ & $8.1(21)$ \\
C3 & $0.2039(22)$ & $0.3879(21)$ & $0.1309(17)$ & $6.1(18)$ \\
C4 & $0.0072(22)$ & $0.3345(25)$ & $0.0904(20)$ & $7.8(19)$ \\
C5 & $0.1060(22)$ & $0.2035(28)$ & $0.1919(21)$ & $9.5(23)$ \\
C6 & $0.3123(23)$ & $0.3454(28)$ & $0.4423(20)$ & $7.9(9)$ \\
C7 & $0.4004(37)$ & $0.4179(38)$ & $0.4083(30)$ & $4.1(11)$ \\
C8 & 0.4654 & 0.3665 & 0.3475 & 7.9 \\
NC1 & $0.1832(18)$ & $0.3378(21)$ & $0.3144(16)$ & $6.5(7)$ \\
NC2 & $0.2402(18)$ & $0.3352(20)$ & $0.3747(15)$ & $5.7(6)$ \\
O1 & $0.1492(15)$ & $0.6890(18)$ & $0.1598(15)$ & $9.6(15)$ \\
O2 & $-0.1276(19)$ & $0.5394(23)$ & $0.0785(13)$ & $14.6(20)$ \\
O3 & $0.2702(16)$ & $0.4156(17)$ & $0.0880(12)$ & $7.9(13)$ \\
O4 & $-0.0427(17)$ & $0.3274(29)$ & $0.0281(14)$ & $14.9(23)$ \\
O5 & $0.1157(17)$ & $0.1105(19)$ & $0.1911(17)$ & $11.8(17)$ \\
\hline
\end{tabular}

${ }^{a} B_{\text {iso }}$ is the mean of the principal axes of the thermal ellipsoid.
TABLE 5. Selected bond distances and angles for $\mathrm{Os}_{3}(\mathrm{CO})_{10}(\mathrm{CNPr})$ (NCMe) (1b)

\begin{tabular}{|c|c|c|c|}
\hline \multicolumn{4}{|l|}{ Bond Distances $(\AA)$} \\
\hline $\operatorname{Os}(1)-\operatorname{Os}(2)$ & $2.8761(11)$ & $\operatorname{Os}(2)-N(C 1)$ & $2.154(24)$ \\
\hline $\operatorname{Os}(1)-O s(2)$ & $2.8761(11)$ & $C(1)-O(1)$ & $1.16(4)$ \\
\hline$O s(1)-C(1)$ & $1.90(3)$ & $C(2)-O(2)$ & $1.05(4)$ \\
\hline $\mathrm{Os}(1)-\mathrm{C}(1)$ & $1.90(3)$ & $C(3)-O(3)$ & $1.14(3)$ \\
\hline $\mathrm{Os}(1)-\mathrm{C}(2)$ & $2.03(3)$ & $C(4)-O(4)$ & $1.14(4)$ \\
\hline $\mathrm{Os}(1)-\mathrm{C}(2)$ & $2.03(3)$ & $C(5)-O(5)$ & $1.18(4)$ \\
\hline $\operatorname{Os}(2)-\operatorname{Os}(2)$ & $2.8662(23)$ & $C(6)-C(7)$ & $1.57(6)$ \\
\hline$O s(2)-C(3)$ & $1.90(3)$ & $C(6)-N(C .2)$ & $1.40(4)$ \\
\hline $\operatorname{Os}(2)-C(4)$ & $1.93(3)$ & $C(7)-C(8)$ & $1.41(5)$ \\
\hline $\mathrm{Os}(2)-\mathrm{C}(5)$ & $1.77(4)$ & $N(C 1)-N(C 2)$ & $1.18(3)$ \\
\hline \multicolumn{4}{|l|}{ Bond Angles (deg) } \\
\hline $\operatorname{Os}(2)-O s(1)-O s(2)$ & $59.77(5)$ & $\mathrm{Os}(2)-\mathrm{Os}(2)-\mathrm{C}(3)$ & $162.2(8)$ \\
\hline $\mathrm{Os}(2)-\mathrm{Os}(1)-\mathrm{C}(1)$ & $99.0(9)$ & $\mathrm{Os}(2)-\mathrm{Os}(2)-\mathrm{C}(4)$ & $89.2(8)$ \\
\hline Os(2)-Os(1)-C(1) & $158.8(9)$ & $\operatorname{Os}(2)-O s(2)-C(5)$ & $97.2(9)$ \\
\hline $\mathrm{Os}(2)-\mathrm{Os}(1)-\mathrm{C}(2)$ & $91.3(10)$ & $\mathrm{Os}(2)-\mathrm{Os}(2)-\mathrm{N}(\mathrm{C} 1)$ & $91.5(6)$ \\
\hline $\mathrm{Os}(2)-\mathrm{Os}(1)-\mathrm{C}(2)$ & $90.1(8)$ & $C(3)-O s(2)-C(4)$ & $92.6(11)$ \\
\hline $\mathrm{Os}(2)-\mathrm{Os}(1)-\mathrm{C}(1)$ & $158.8(9)$ & $C(3)-O s(2)-C(5)$ & $100.6(12)$ \\
\hline $\mathrm{Os}(2)-\mathrm{Os}(1)-\mathrm{C}(1)$ & $99.0(9)$ & $\mathrm{C}(3)-\mathrm{Os}(2)-\mathrm{N}(\mathrm{C} 1)$ & $88.1(10)$ \\
\hline $\operatorname{Os}(2)-\operatorname{Os}(1)-C(2)$ & $90.1(8)$ & $C(4)-O s(2)-C(5)$ & $89.3(14)$ \\
\hline $\operatorname{Os}(2)-O s(1)-C(2)$ & $91.3(10)$ & $\mathrm{C}(4)-\mathrm{Os}(2)-\mathrm{N}(\mathrm{C} 1)$ & $175.7(12)$ \\
\hline$C(1)-O s(1)-C(1)$ & $102.1(13)$ & $C(5)-O s(2)-N(C 1)$ & $86.4(13)$ \\
\hline $\mathrm{C}(1)-\mathrm{Os}(1)-\mathrm{C}(2)$ & $89.8(12)$ & $\mathrm{Os}(1)-\mathrm{C}(1)-\mathrm{O}(1)$ & $178(3)$ \\
\hline$C(1)-O s(1)-C(2)$ & $89.1(13)$ & $\mathrm{Os}(1)-\mathrm{C}(2)-\mathrm{O}(2)$ & $175(3)$ \\
\hline$C(1)-O s(1)-C(2)$ & $89.1(13)$ & $\mathrm{Os}(2)-\mathrm{C}(3)-\mathrm{O}(3)$ & $176.9(23)$ \\
\hline$C(1)-O s(1)-C(2)$ & $89.8(12)$ & $\mathrm{Os}(2)-\mathrm{C}(4)-\mathrm{O}(4)$ & $178(3)$ \\
\hline$C(2)-O s(1)-C(2)$ & $178.3(13)$ & $O s(2)-C(5)-O(5)$ & $178(3)$ \\
\hline $\operatorname{Os}(1)-\operatorname{Os}(2)-\operatorname{Os}(2)$ & $60.11(4)$ & $C(7)-C(6)-N(C 2)$ & $109(3)$ \\
\hline $\operatorname{Os}(1)-\operatorname{Os}(2)-C(3)$ & $102.1(8)$ & $C(6)-C(7)-C(8)$ & $113(3)$ \\
\hline $\operatorname{Os}(1)-O s(2)-C(4)$ & $92.0(9)$ & $\operatorname{Os}(2)-N(C 1)-N(C 2)$ & $175.2(21)$ \\
\hline $\mathrm{Os}(1)-\mathrm{Os}(2)-\mathrm{C}(5)$ & $157.2(9)$ & $C(6)-N(C 2)-N(C 1)$ & $172(3)$ \\
\hline $\mathrm{Os}(1)-\mathrm{Os}(2)-\mathrm{N}(\mathrm{C} 1)$ & $92.0(7)$ & & \\
\hline
\end{tabular}

$4 \times 15 \AA^{3}$ ) leaving a large space available for the totally disordered CNPr and NCMe (8 non-H atoms total).

\section{Experimental section}

\subsection{Crystallographic structure determination}

The synthesis of $\mathrm{Os}_{3}(\mathrm{CO})_{10}(\mathrm{CNPh})(\mathrm{NCMe})$ and $\mathrm{Os}_{3}(\mathrm{CO})_{10}(\mathrm{CNPr})(\mathrm{NCMe})$ follows the reaction of $\mathrm{Os}_{3}(\mathrm{CO})_{12}$ with phosphine imides, then oxidative decarbonylation by $\mathrm{Me}_{3} \mathrm{NO}$ in the presence of $\mathrm{MeCN}$ as detailed elsewhere [5]. Crystals of both $\mathrm{Os}_{3}$ $(\mathrm{CO})_{10}(\mathrm{CNPh})(\mathrm{NCMe})(\mathbf{1 a})$ and $\mathrm{Os}_{3}(\mathrm{CO})_{10}(\mathrm{CNPr})$ (NCMe) (1b) were obtained from slow evaporation of hexane $/ \mathrm{CH}_{2} \mathrm{Cl}_{2} / \mathrm{MeCN}$ solutions at $-5^{\circ} \mathrm{C}$. Specimens of suitable quality were mounted in a glass capillary for precise determination of cell constants and intensity data collection was made on an Enraf-Nonius CAD-4 diffractometer using graphite-monochromated $\mathrm{MoK} \alpha$ radiation $(\lambda=0.70930 \AA)$ with $\theta-2 \theta$ scan mode. Unit cells were determined and refined using 25 randomly 
selected reflections obtained using the CAD-4 automatic search, centre, index and least-squares routines. Space groups were determined from the systematic absences observed during data collection. Systematic absences in the diffraction data of 1a and 1b unambiguously established the space groups as $P 2_{1} / m$ and $P b c n$, respectively. An empirical absorption correction based on azimuthal scans of three reflections was applied to each of the data sets. The structures were solved by a combination of heavy-atom methods and difference syntheses, and was refined by a full-matrix least-squares procedure in which the function $\Sigma \omega \Delta^{2}$ was minimized where $\Delta=\left\|F_{o}|-| F_{c}\right\|$ and $\omega$ is the counting statistical weight applied to each reflection. Refinement of 1a: The NCMe group and $\mathrm{C}(6) \mathrm{O}(6)$ ligand were disordered across a mirror plane, the disorder being 50/50 as required crystallographically. The atoms of $\mathrm{C}(6), \mathrm{O}(6), \mathrm{N}(2), \mathrm{C}(12)$, and $\mathrm{C}(13)$ could be clearly resolved and independently refined anisotropically. Refinement of the disordered model led to convergence with $R=0.037, R_{w}=0.029$ and $\mathrm{GOF}=2.50$ for data with $\left|F_{o}\right|>2 \sigma\left(\left|F_{o}\right|\right)$. Removal of the crystallographic mirror, i.e., using space group $P 2_{1}$ instead of $P 2_{1} / m$ led to singularity such that the attempted refinement was not possible. Refinement of $1 \mathbf{b}$ : The molecule sits on a crystallographic two-fold axis such that the ligands $\mathrm{NCMe}$ and $\mathrm{CNPr}$ are symmetrically related in equivalent occupancy. The two donating atoms of ligands NCMe and CNPr were accordingly refined with one common set of atomic coordinates and isotropic thermal parameters, with $50 \mathrm{~N} / 50 \mathrm{C}$ composite scattering factor. The extending chain- $\mathrm{C}(6)$, $\mathrm{C}(7)$, and $\mathrm{C}(8)$ - of NCMe and CNPr were quite resistive to the analysis, too. The atoms of $C(6)$ and $C(7)$ were refined isotropically. The atom of $\mathrm{C}(8)$ was located and isotropically fixed. The reduction from $\mathrm{Pbcn}$ to lower symmetric space groups produced poorer results. Crystallographic data for $1 \mathrm{a}$ and $1 \mathrm{~b}$ are shown in Table 1. Final atomic coordinates, isotropic thermal parameters, and selected bond distances and bond angles for the two complexes are listed in Tables 2-5. All the data processing was carried out on a Microvax 3600 using the NRCC SDP program [9].

\section{Acknowledgment}

We thank the National Science Council of Taiwan for financial support.

\section{Supplementary material}

Tables of atomic coordinates, crystal and intensity collection data, anisotropic thermal parameters, bond lengths and angles (8 pages), and tables of structure factors (16 pages) are available from the authors.

\section{References}

1 K. Yoon and G. Parkin, Inorg. Chem., 31 (1992) 1656.

2 (a) M.R. Churchill, J.C. Fettinger, L.A. Buttrey, M.D. Barkan and J.S. Thompson, J. Organomet. Chem., 340 (1988) 257; (b) K.R. Durbar and S.C. Haefner, Inorg. Chem., 31 (1992) 3676; (c) W.M. Rees, M.R. Churchill, Y.J. Li and J.D. Atwood, Organometallics, 4 (1985) 1162; (d) J.C. Fettinger, M.R. Churchill, K.A. Bernard and J.D. Atwood, J. Organomet. Chem., 340 (1988) 377; (e) J.A. Mcginnety, R.J. Doedens and J.A. Ibers, Inorg. Chem., 6 (1967) 2243; (f) S.J. La Placa and J.A. Ibers, J. Am. Chem. Soc., 87 (1965) 2581.

3 (a) E. Carmona, J.M. Marin, M.L. Poveda, J.L. Atwood and R.D. Rogers, Polyhedron, 2 (1983) 185; (b) G.S. Girolami, J.E. Salt, G. Wilkinson, M. Thornton-Pett and M.B. Hursthouse, J. Am. Chem. Soc., 105 (1983) 5954; (c) J.E. Salt, G.S. Girolami, G. Wilkinson, M. Motevalli, M. Thornton-Pett and M.B. Hursthouse, J. Chem. Soc., Dalton Trans., (1985) 685; (d) B.R. Davis and J.A. Ibers, Inorg. Chem., 10 (1971) 578.

4 K.W. Chiu, R.A. Jones, G. Wilkinsom, A.M.R. Galas, M.B. Hursthouse and K.M.A. Malik, J. Chem. Soc., Dalton Trans., (1981) 1204.

5 (a) K.L. Lu, C.J. Su, Y.W. Lin, H.M. Gau and Y.S. Wen, Organometallics, 11 (1992) 3932; (b) K.L. Lu, H.J. Chen, P.Y. Lu, S.Y. Li, F.E. Hong, S.M. Peng and G.H. Lee, Organometallics, in press.

6 P.A. Dawson, B.F.G. Johnson, J. Lewis, J. Puga, P.R. Raithby and M.J. Rosales, J. Chem. Soc., Dalton Trans., (1982) 233.

7 (a) P. Legzdins, G.B. Richter-Addo, B. Wassink, F.W.B. Einstein, R.H. Jones and A.C. Willis, J. Am. Chem. Soc., 111 (1989) 2097; (b) X. Bu, I. Cisarova, P. Coppens, B. Lederle and M.J. Naughton, Acta Cryst., C48 (1992) 516.

8 M.R. Churchill and B.G. DeBoer, Inorg. Chem., 16 (1977) 878.

9 E.J. Gabe, F.L. Lee and Y. Le Page, in G.M. Sheldrick, C. Krüger and R. Goddard (eds.), Crystallographic Computing 3; Data Collection, Structure Determination, Proteins, and Databases, Clarendon Press, Oxford, UK, 1985, pp. 167-174. 